\title{
Familial hidradenitis suppurativa: evidence in favour of single gene transmission
}

\author{
J S FITZSIMMONS*, E M FITZSIMMONS*, AND G GILBERT \\ From the *Clinical Genetic Service and †Department of Photography, City Hospital, \\ Hucknall Road, Nottingham NG5 IPB.
}

SUMMARY The three English families in this report have a total of 21 members ( 16 females and five males) suffering from chronic hidradenitis suppurativa. In family $A$ the condition is associated with acne conglobata and there is vertical transmission of the disorder through three generations. In the others there is no associated cystic acne, but all those affected had a history of acne vulgaris with comedone formation, and the condition has been confirmed in two generations. It is likely that a male in an earlier generation in family B suffered from the disease and affected sibs in family $\mathrm{C}$ were born to different fathers. The familial aggregation and number of affected subjects suggests a single gene disorder and the pattern of transmission is consistent with autosomal dominant inheritance. Males and females are affected in successive generations and there is the anticipated variation in clinical severity in those suffering from the condition.

Hidradenitis suppurativa ('apocrinitis') is a clinically striking but apparently uncommon chronic inflammatory condition of the skin and subcutaneous tissues in areas containing apocrine sweat glands. ${ }^{12}$ These are located deep in the dermis and are closely associated with the pilosebaceous organs. Although widely scattered over the body surface these glands are concentrated mainly in the axillae, perianal regions, lower back, and in and around the breasts. The disease has been recognised for many years, but the basic cause of the disorder remains obscure and it has been classified with acne conglobata and dissecting cellulitis of the scalp as the follicular occlusive triad. ${ }^{3}$ Acne conglobata with comedones, cysts, and abscesses of back, chest, and buttocks appears to be the most common single lesion of this group, but some subjects develop an associated hidradenitis and a few suffer from all three conditions. Hidradenitis suppurativa occurs in both sexes, but appears to be more common in females, and the age of onset varies from adolescence to early or mid adult life, although acne conglobata can be seen earlier. Lesions of hidradenitis occur in the axillae, particularly in the female, but perianal and genital lesions occur in both sexes and are more resistant to treatment. The condition is thought to start in the pilosebaceous follicles with poral occlusion consequent on comedone formation.

Received for publication 15 November 1983. Accepted for publication 9 December 1983.
Secondary obstruction and bacterial infection of the adjacent apocrine glands then occurs, and the swollen and usually painful glands rupture onto the surface of the skin or laterally into the subcutaneous tissues. This may produce infection of other glands with cellulitis, sinus formation, and subcutaneous tunnelling. The lesions initially heal with little residual scarring, but after some years there is usually considerable fibrosis and induration. The disease, when severe, is a burdensome one and the relapsing and chronic nature of the infection may have considerable psychological effects. Fortunately, the general health of the majority of patients remains good but a number have been found to have associated anaemia, ${ }^{4}$ and occasional fatalities from generalised septicaemia have been reported. Fistula formation with involvement of pelvic organs has also been described in severe hidradenitis affecting the perianal region, ${ }^{5}$ and the chronic skin lesions predispose to neoplastic change in a small number of patients. ${ }^{6}$ A non-specific spondyloarthropathy occurs in some affected subjects, ${ }^{7}$ usually those with an associated acne conglobata. The disease normally becomes quiescent after many years of activity and in most affected females the severity of the condition declines at or following the menopause.

A number of precipitating factors have been described. These include the use of depilating agents, deodorants, pressure from clothing, poor hygiene, and others. Similar acute lesions of the apocrine 
glands can be produced experimentally by the application of adhesive tape to the axillae. Obesity appears to be a common finding in females and diabetes has been described in occasional patients. However, although endocrine factors are considered to be of importance in acneiform lesions, their role in hidradenitis is less obvious and no consistent endocrine abnormality has been reported. Females with the disease usually notice a worsening of the condition just before and early in menstruation, probably as a consequence of increase in activily of the apocrine glands at this time. Pregnancy alleviates the disorder in some, but not in others.

\section{Family studies}

FAMILY A (FIG 1)

III.I2. The proband was born on 23.7.56 and attended the Clinical Genetic Service in 1983 requesting genetic counselling in view of the familial chronic skin disease. She started with cystic acne on her buttocks and back at the age of 5 and at varying intervals over the following years she developed swellings and abscesses in her axillae, groins, and breasts, the typical manifestations of hidradenitis suppurativa. Some of the inflammatory lesions required incision, others discharged spontaneously, and despite various courses of antibiotics the condition persisted. Her general health has remained good, but she was sufficiently distressed by the skin complaint to decide not to have children if there was a significant risk to them.

II.5. The mother of III.12 was born on 10.1.37 and also started with acne conglobata about the age of 5 . The first lesions were noted on her buttocks but eventually also involved her back, and like her daughter she subsequently developed hidradenitis suppurativa in her axillae and groins (figs 2 and 3 ). Despite long term treatment with oxytetracycline the lesions recurred and many required surgical treatment. She had no problems during her pregnancies, but when breast feeding she had particularly troublesome inflammatory swellings in her axillae and breasts and at one stage was found to be anaemic.

III.13. This patient was born on 15.7.57 and started with an inflammatory lesion between her breasts and in the skin over the lower spine at the age of 19. To date these have not required incision but regularly discharge spontaneously. Her axillae have not been affected.

II.6. This patient was born on 4.6 .40 and also started with cystic acne when she was 5 or 6 years of
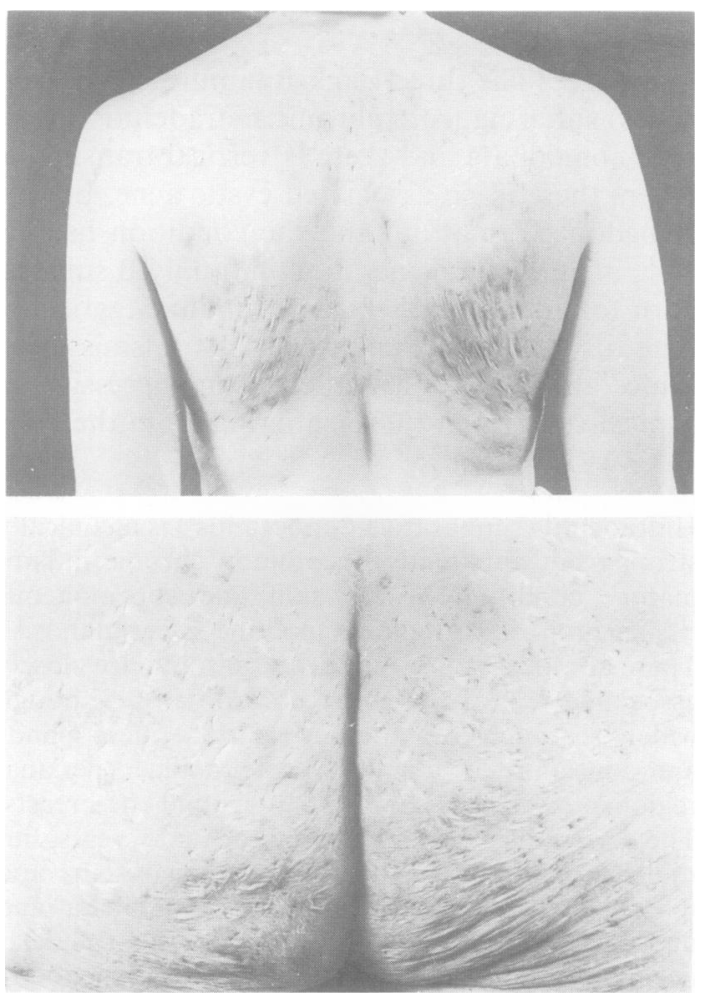

FIC; 2 Typical healed lesions of acne conglohata on back and huttocks in II.5, family A.

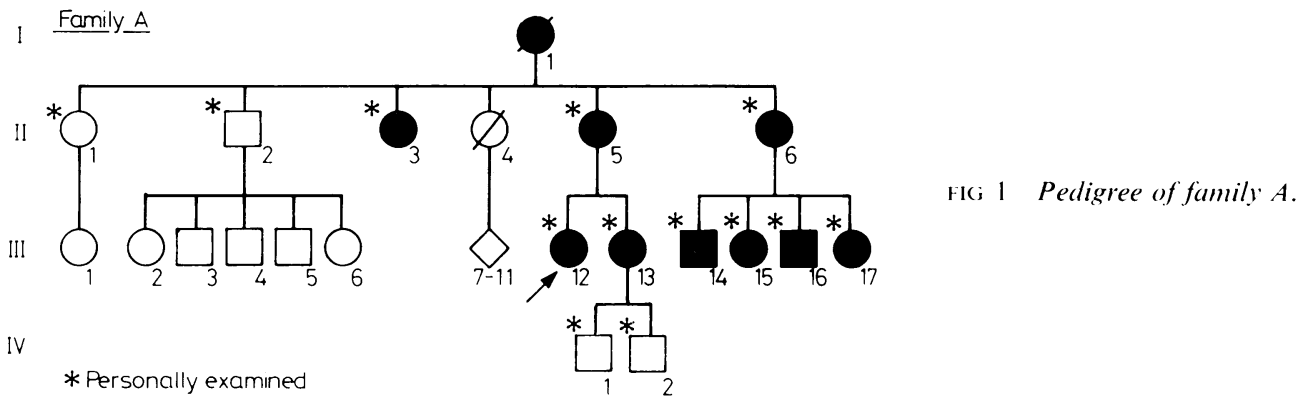




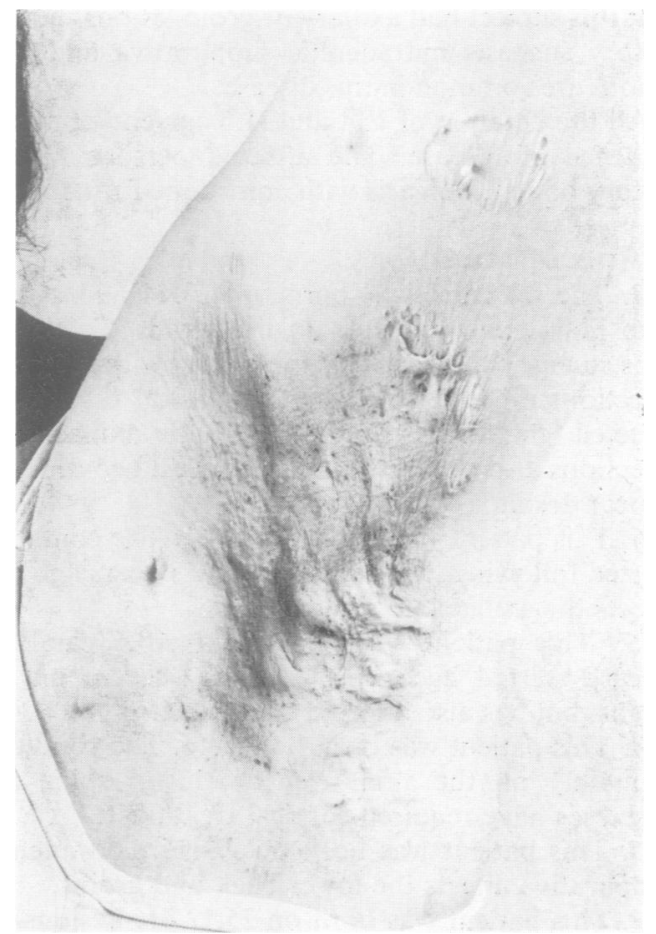

FIG 3 Typical lesions of hidradenitis with induration, scarring, subcutaneous tunnelling, and marked comedone formation in II.5, family A. The perianal and genital regions are severely affected. age. The natural history and distribution of the lesions were similar to those of her sister and niece, but in addition she has recurrent abscesses on the back of her neck. All four of her children are affected. Their disease also started early in life, certainly before their teens, and all continue to have problems. III.14. This patient was born on 29.6.64 and has severe lesions on the thighs, lower trunk, arms, axillae, and buttocks (fig 4). The condition started early, but worsened considerably at the age of 12 or 13 .

III.15. This patient was born on 17.9.65 and has lesions primarily in the axillae and groins with some on the back of the neck. They are much less severe than those of her older brother.

III.16. This patient was born on 6.7.68 and has typical lesions in the axillae, back of neck, buttocks, and legs.

III.17. This patient was born on 22.6 .70 and has lesions in the groins and minimally in the axillae.

II.3. This patient was born on 20.7.25 and developed low back lesions which required surgical incision. At the time of examination (17.10.83) she had two healing inflammatory swellings on her lower abdomen. Both axillae appeared normal.

FAMILY B (FIG 5)

II.3. This patient started with axillary lesions in his teens. These have recurred regularly over the years and he has had as many as 50 in total. He had no lesions elsewhere and was not seriously affected by acne as a teenager. He has no evidence of acne
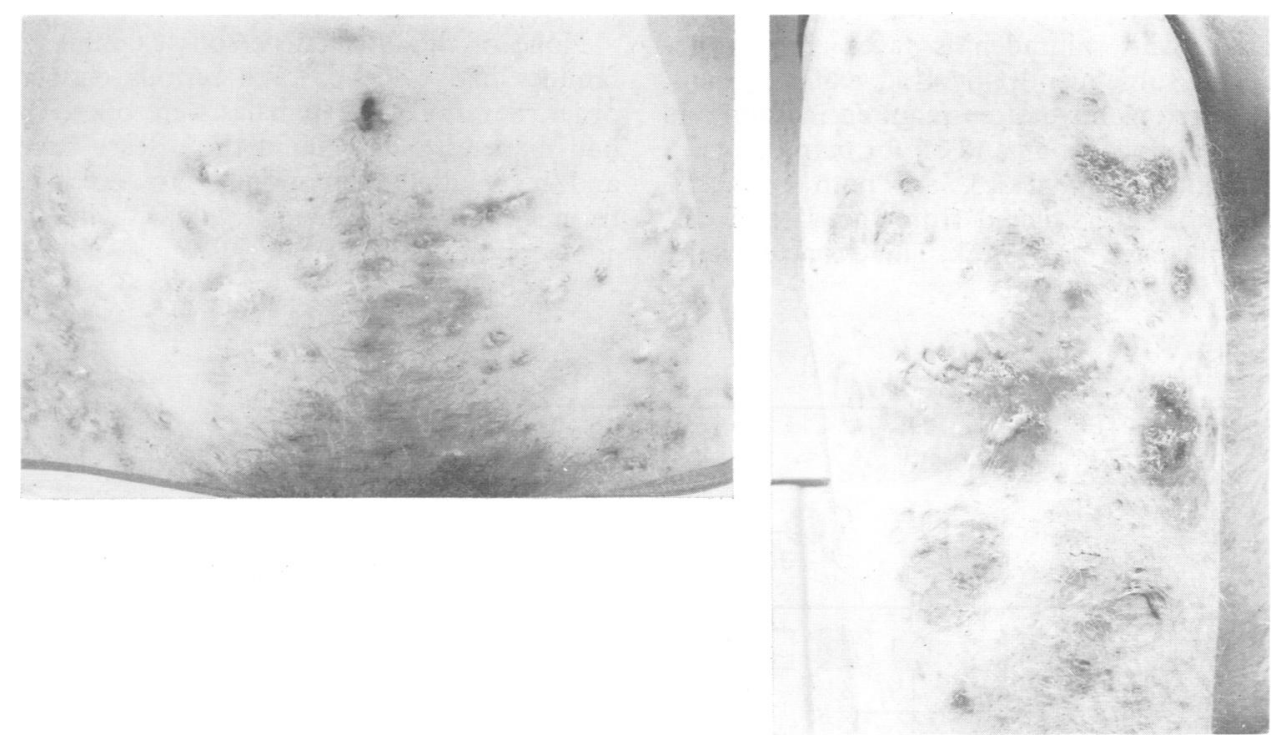

FIG 4 III.14, family A; severe lesions of hidradenitis on thighs and lower abdomen. II.7, family C, with similar lesions on thighs. 


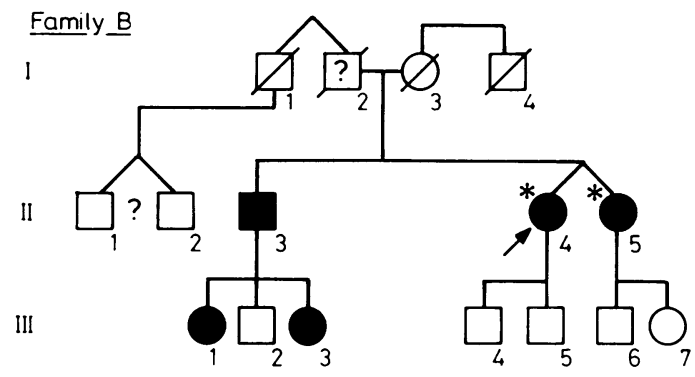

FIG 5 Pedigree of family $B$.

conglobata but still develops comedones on the face and suffered from acne vulgaris in early life.

III.1. This patient started with groin and vulval lesions in her teens. The vulval lesions have recurred but usually heal without surgical treatment.

III.3. Axillary lesions were noted in this patient at the age of 17 , and she subsequently developed painful swellings on her breasts and a number in the groin and vulval area. To date these have not required surgical incision and have regressed spontaneously.

II.4. The proband was born on 6.3.42. The first abscess occurred in the breast at the age of 20 and since then further lesions have occurred on the breasts, perineum, and base of the spine. A number of these required surgical incision and despite antibiotics have continued to recur.

II.5. This identical twin to II.4 was born on 7.3.42 and started with hidradenitis aged 23 on her breasts. This subsequently involved axillae, groins, and back. Lesions have often required incision and she has had antibiotics on and off for many years.

The parents, I.2 and I.3, are both dead. II.3 remembers that I.2 suffered from 'boils' and carbuncles on the back of the neck. There is no evidence that this subject had axillary or groin lesions, but the history suggests hidradenitis suppurativa and neck lesions are common in this disease.

All the children of II.4 and II.5 currently have no evidence of disease. The affected persons have a history of acne vulgaris with comedone formation.

\section{FAMILY C (FIG 6)}

II.3. The proband was born on 1.3.44 and started with hidradenitis aged 14 in the groins. There was subsequent involvement of the axillae with recurrent infections eventually leading to surgical removal of infected glandular tissue from both axillae. The infections also occurred in the groin and continue to appear despite regular antibiotics.

II.6. This patient was born on 6.7.61. The condition started following the first pregnancy 3 years ago and affects the axillae and groins.

III.7. This patient was born on 14.10 .63 and the disease started aged 19. It is very severe on the thighs, but has also affected the axillae.

II.4. This patient was born on 7.5.46. The condition is mainly on the skin over the lower spine and abscesses have required surgical treatment.

II.5. This patient was born on 9.3.56. Hidradenitis suppurativa affects the lower back and groins.

II.2. This patient was born on 25.12.41. Lesions are mainly on the back and groin.

I.2, the mother of the affected persons, married twice. There is no definite confirmation that she suffered from hidradenitis and she died at the age of 55 from carcinoma of the lung. However, she is the link between the affected sibs born to different fathers.

None of the affected persons examined in these families had evidence of a serious endocrine disorder. Several of the females were obese, but none had diabetes. Most found the disease burdensome and had suffered emotionally to varying degrees from its effects. All had acne vulgaris with prominent comedones.

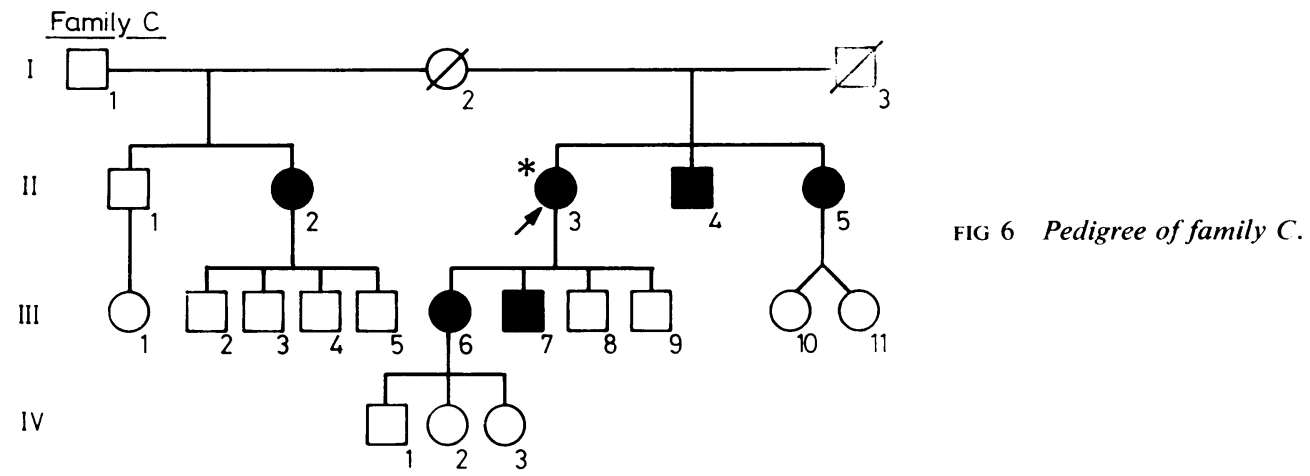




\section{Discussion}

Although hidradenitis suppurativa is listed as one of the poral occlusive disorders, the primary cause of the poral occlusion is obscure and there is no firm evidence that the apocrine glands in these patients are anatomically different from those in persons without the disease. Sulzberger and $\mathrm{Baer}^{8}$ refer to a possible abnormality of the glandular ducts in familial hidradenitis, but do not support their comments with further details or references. Dermatologists appear to accept that the occlusion occurs initially in the pilosebaceous apparatus secondary to comedone formation in the outlet of the sebaceous gland (fig 3 ). This in turn produces obstruction of the apocrine gland because of the close proximity of these two structures. The formation of comedones is important in the production of acne vulgaris, but although common in hidradenitis, some other factor or factors must be important. Many otherwise normal subjects develop acne and acneiform lesions particularly during adolescence, but only a small number develop chronic hidradenitis suppurativa. Infection, usually with Staphylococcus aureus, is obviously important and the recurrence of the lesions clearly raises the possibility of a generalised defect in host defence mechanisms. However, patients with this disease do not seem to be more prone to infections in other organs and most evidence confirms that infection is a secondary event and does not have a primary role. Extensive investigations of subjects II.4 and II.5 in family B revealed no abnormality of their immunological system and no host defence failure was found in seven patients investigated by Dvorak et al. ${ }^{9}$ There have been no reports of any abnormality of apocrine gland secretion in this condition and the distribution and control of the glands appears to be normal. Despite the occurrence of anaemia in some patients, this is also considered to be a secondary rather than a primary factor. The anaemia may be normochromic or hypochromic and is presumed to have the same origin as the anaemia associated with other types of chronic infection. There have been no reports of amyloidosis or significant hypoproteinaemia in patients with severe and relapsing disease.

The bulk of published reports on hidradenitis has been concerned with treatment and there has been little or no attention paid to family details. Knaysi et al ${ }^{10}$ noted a family history in three of 18 patients specifically questioned. Cockayne ${ }^{11}$ suggested that acne vulgaris behaved as a simple dominant, but Stokes and King ${ }^{12}$ considered heritable factors in acne to be complex and not appearing to follow a simple Mendelian distribution. The familial nature of hidradenitis is occasionally mentioned in published reports, but pedigree information is lacking and further detailed family studies are necessary if a genetic basis for the disorder is to be confirmed. The disease is probably more common than suspected and some persons may be assumed to have recurrent perianal abscesses or simply recurrent 'boils'. It is also likely that less severely affected persons have been overlooked in the past. Several subjects in family B had vulval lesions only and had been reluctant to seek medical attention for them over the years. The wide variation in clinical severity and age of onset of this disease needs to be appreciated.

The authors wish to thank Dr B R Allen, Consultant Dermatologist, and Dr P J Toghill, Consultant Physician, Queen's Medical Centre, Nottingham, for referring two of the families in this report. We are also grateful to Miss Barbara A Beasley for typing the manuscript.

\section{References}

1 Brunsting HA. Hidradenitis and other variants of acne. Arch Dermatol 1952;65:303-15.

2 Brunsting HA. Hidradenitis suppurativa; abscess of the apocrine sweat glands. Arch Dermatol 1939;39:108-20.

3 Self SJ, Montes LF. Follicular occlusion triad. South Med J 1970;63:156-60.

4 Gordon SW. Hidradenitis suppurativa: a closer look. J Natl Med Assoc 1978;70:339-43.

5 Ching CC, Stahlgren LH. Clinical review of hidradenitis suppurativa. Management of cases with severe perianal involvement. Dis Colon Rectum 1965;8:349-52.

- Humphrey LJ, Playforth H, Leavell UW, et al. Squamous cell carcinoma arising in hidradenitis suppurativum. Arch Dermatol 1969;100:59-62.

7 Rosner IA, Richter DE, Huettner TL. Spondyloarthropathy association with hidradenitis suppurativa and acne conglobata. Ann Intern Med 1982;97:520-5.

8 Sulzberger MD, Baer RL. Treatment of pyodermas (common pus-forming infections of the skin. In: The 1950 yearbook of dermatology and syphilology. Chicago: Yearbook Medical Publishers, 1951:46.

- Dvorak VC, Root RK, MacGregor RR. Host-defense mechanisms in hidradenitis suppurativa. Arch Dermatol 1977;113:450-3.

10 Knaysi GA, Cosman B, Crikelair GF. Hidradenitis suppurativa. JAMA 1968;203:19-22.

11 Cockayne EA. Inherited abnormalities of the skin and its appendages. London: Oxford University Press, 1933:351.

12 Stokes JH, King AD. Acne vulgaris: heredity in the etiologic background. Arch Dermatol 1932;26:456.

Correspondence and requests for reprints to $\mathrm{Dr} \mathrm{J} S$ Fitzsimmons, Clinical Genetic Service, City Hospital, Hucknall Road, Nottingham NG5 1 PB. 